\title{
EVALUATION OF CONE BEAM CT IN DIAGNOSIS OF RAT'S TEMPOROMANDIBULAR JOINT ARTHRITIS
}

\author{
Sahar M. Samir*, Mona M. Abou El-Fotouh* and Walaa M. Hamed*
}

\begin{abstract}
Objective: To find out the diagnostic performance of cone beam CT in detection of chemically induced arthritic changes of rats' condyles using the histological sections as a gold standard.

Methods: 40 male albino rats were divided into study and control groups each contains 20 rats. Arthritis was induced into the right temporomandibular joint (TMJ) of the study group using complete Freund's adjuvant (CFA) inflammatory material. All rats were incubated for 21 days then all rats were anesthetized, sacrificed then imaged using Planmeca ProMax 3D Proface (Planmeca Oy, Finland) cone beam CT. Heads were immediately prepared for histological sections using Haematoxylin and Eosin (H\&E) stain. Condylar osseous changes were evaluated by both methods. Chi square test was used to compare between CBCT and histological findings. Sensitivity, specificity, accuracy, positive predictive value (PPV) and negative predictive value (NPV) determined CBCT diagnostic performance using histological findings as a gold standard.
\end{abstract}

Results: There was a statistically significant difference between CBCT and histological findings while they were totally matching in condylar erosion findings. Condylar flattening couldn't be detected histologically and the synovial proliferation was a histological result only. CBCT diagnostic accuracy was $95.29 \%$, the sensitivity was $93 \%$, the specificity was $100 \%$, the NPV was $86 \%$ and the PPV was $100 \%$.

Conclusion: High diagnostic performance of CBCT in detection of rat's condylar arthritis.

KEYWORDS: Arthritis; temporomandibular joint (TMJ); cone beam CT; complete Freund's adjuvant (CFA)

\section{INTRODUCTION}

Temporomandibular disorders (TMD) describe any articular or muscular problem of the jaw joint. TMJ osteoarthritis is caused by alternating degenerative and regenerative episodes causing structural changes of the joint components such as erosion, flattening, osteophytes or reduced joint space. ${ }^{1}$

\footnotetext{
* Department of Oral Radiology, School of Dentistry, University of Ain Shams, Cairo, Egypt
} 
Diagnostic imaging of TMD is very challenging due to complexity of TMJ structure and superimposition from base of the skull ${ }^{2}$ which mandate the use of 3D imaging. Advantages of cone beam $\mathrm{CT}$ regarding low dose and cost, fast scan time, easy software and small $\mathrm{FOV}^{3}$ put $\mathrm{CBCT}$ into competition with other diagnostic modalities.

Therefore we thought it is worthwhile to explore the diagnostic performance of CBCT in detection of artificially (chemically) induced TMJ arthritis using rats as an animal model.

\section{MATERIALS AND METHODS}

Forty male Albino rats weighing between 200250 grams were divided into two groups; a control group of 20 rats used as a reference for the normal radiographic and histologic picture of rat's TMJ and a study group of 20 rats used as animal models for TMJ arthritis.

This study was approved from Faculty of Dentistry Ain Shams University research ethics committee (FDASU-REC). Arthritis was induced by intra-articular injection (50 microns) into the right TMJ of complete Freund's adjuvant (CFA, purchased from Sigma) via a carefully positioned tip of a 26 gauge needle. The CFA was suspended in an oil/saline (1:1) emulsion. ${ }^{4}$

At the end of the housing period (21 days), each rat of both study and control groups was anesthetized then sacrificed separately by cervical dislocation and their heads were immediately fixed at $10 \%$ buffered formalin solution. Getting rid of sacrificed rats' bodies was in the incinerator of Cairo University Hospital.

CBCT examination was performed using Planmeca ProMax 3D Proface (Planmeca Oy, Finland) and Planmeca Romexis Viewer 3.0.0.R. was used to view images in axial, coronal and sagittal planes. Two oral and maxillofacial radiologists with five years experience in radiographic interpretation performed the radiographic image analysis. The images were rechecked by both observers to confirm the intra-observer reliability. The recorded findings were those agreed at least twice. Criteria for evaluation of bony changes in the study group were classified into:

1. Condylar surface findings (a) no osseous changes (b) condylar flattening (c) condylar erosion

2. Joint space findings (a) No change (b) obliterated joint space (c) bone to bone contact.

Rats' heads were fixed at $10 \%$ buffered formalin solution for at least one week then placed in $12 \%$ EDTA (Ethylene Diamine Tetra-acetic acid) solution changed every 48 hours for two weeks for decalcification. Sections were performed by microtome (4-6 microns) and stained with Haematoxylin and Eosin (H\&E) stain. The stained histological sections of both study and control groups were captured at a magnification of $40 \mathrm{X}$ using a digital video camera (C5060, Olympus, Japan) which was mounted on a light microscope (BX60, Olympus, Japan). Images were then transferred to the computer system for image analysis.

Three oral histologists with five years experience examined the stained sections for evaluation of histological changes at the tissue level such as new tissue formation, surface irregularity, bone marrow edema or bone erosion. Data obtained from CBCT analysis was subsequently compared with the histological findings as the gold standard. All data were entered into Excel 2007 (Microsoft, Redmond, WA) then statistical analysis was performed to find if there is a significant difference between CBCT and the histological findings.

Observational findings were compared to each other using Chi square test. Sensitivity, specificity, accuracy, positive predictive value (PPV) and negative predictive value (NPV) were used as a measure for CBCT diagnostic performance compared to the histological findings. Intra and inter observer reliability were determined using Chi square test. 


\section{RESULTS}

Results of this study included: Comparison between CBCT and histological observations of osseous changes, Diagnostic performance of CBCT observations and reliability of CBCT observers' readings.

Comparison between CBCT and histological observations of osseous changes (Figures 1-2) are illustrated in Table 1. There was no difference between CBCT and histological observations in condylar erosion findings. On the other hand, statistically significant differences were found with no osseous changes, condylar flattening, decreased joint space and synovial proliferation findings.

Sensitivity, specificity, accuracy, positive predictive value (PPV) and negative predictive value (NPV) determined CBCT diagnostic performance using histological findings as a gold standard are illustrated in Table 2.

On comparing 1 st and 2 nd readings of the CBCT observer (1) there was a statistically significant difference regarding condylar erosion findings. However, no osseous change, condylar flattening and decreased joint space findings showed not statistically significant differences (Table 3). Although, CBCT findings of condylar erosion and no osseous changes differed significantly between 1 st and 2nd readings of the CBCT observer (2),

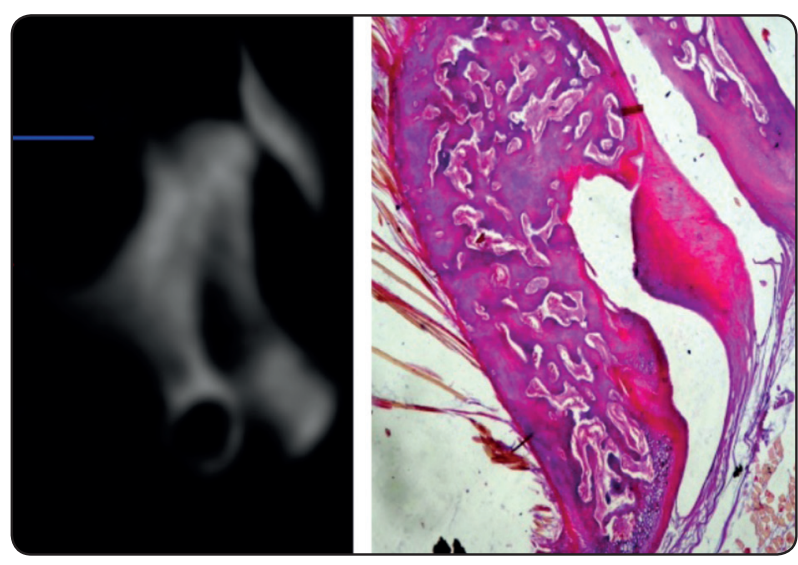

Fig. (2) Condylar erosion on sagittal CBCT and sagittal H\&E sections.

findings of condylar flattening and decreased joint space showed not statistically significant difference (Table 4).

Regarding the 1st readings of both observers; condylar erosion and no osseous changes CBCT findings differed significantly between CBCT observers while, condylar flattening and decreased joint space showed not statistically significant differences between them (Table 5). However, the 2nd readings revealed a statistically significant difference between readings of CBCT observers in condylar erosion findings. However, no osseous change, condylar flattening and decreased joint space findings showed not statistically significant differences (Table 6).
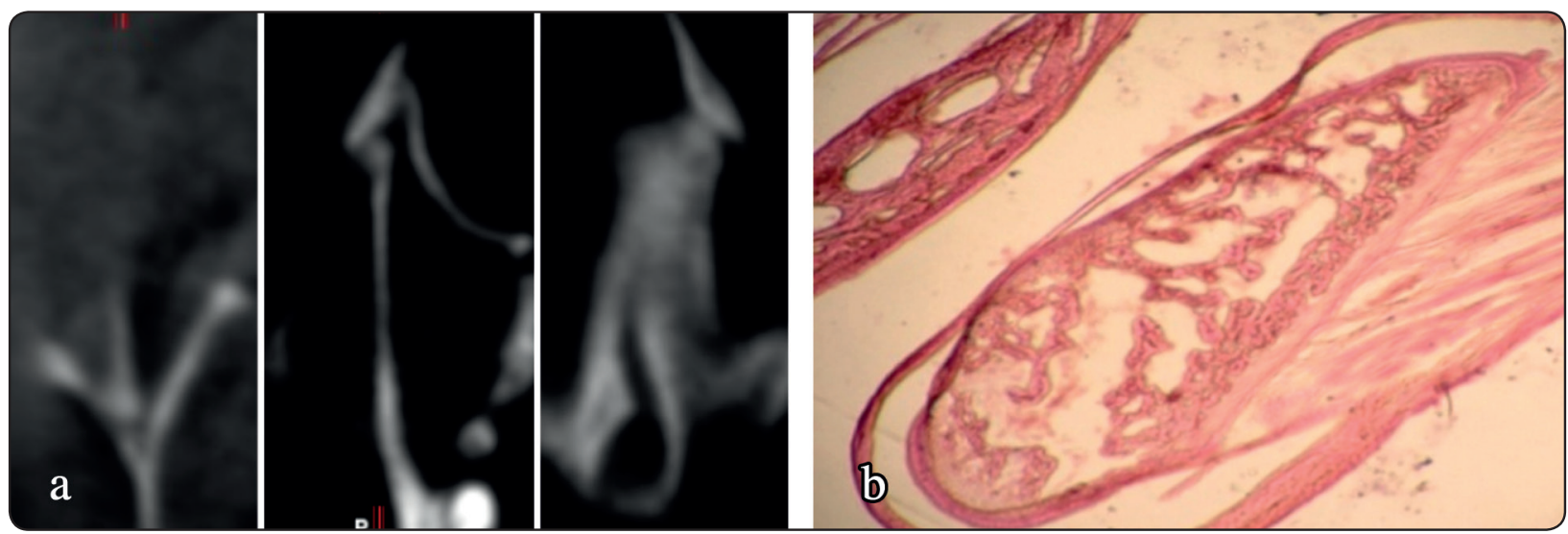

Fig. (1) Case presentation of reduced joint space: (a) axial (left), coronal (middle) and sagittal (right) CBCT sections showing decreased joint space. (b) Sagittal H\&E section of the same joint showing decreased joint space. 
TABLE (1) Comparison between CBCT and histological observations of osseous changes.

\begin{tabular}{|c|c|c|c|c|c|}
\hline \multirow{2}{*}{ Type of observation } & \multicolumn{2}{|c|}{ Histological observations } & \multicolumn{2}{|c|}{ CBCT observations } & \multirow[t]{2}{*}{ P value } \\
\hline & Number & $\%$ & Number & $\%$ & \\
\hline No osseous changes & 0 & 0 & 1 & 5 & $* 0.024$ \\
\hline Condylar erosion & 12 & 60 & 12 & 60 & \\
\hline Condylar flattening & 0 & 0 & 5 & 25 & $* 0.0001$ \\
\hline Decreased Joint Space & 4 & 20 & 2 & 10 & $* 0.024$ \\
\hline Synovial Proliferation & 4 & 20 & 0 & 0 & $* 0.0001$ \\
\hline Total number & 20 & 100 & 20 & 100 & \\
\hline
\end{tabular}

TABLE (2) Diagnostic performance of CBCT

\begin{tabular}{|l|c|c|c|c|c|}
\hline & Sensitivity & Specificity & Accuracy & PPV & NPV \\
\hline CBCT & $93 \%$ & $100 \%$ & $95.29 \%$ & $100 \%$ & $86 \%$ \\
\hline
\end{tabular}

TABLE (3) Intra-observer reliability of the CBCT observer (1):

\begin{tabular}{|c|c|c|c|c|c|}
\hline \multirow{2}{*}{ Types of observations } & \multicolumn{2}{|c|}{$1^{\text {st }}$ reading of observer $(1)$} & \multicolumn{2}{|c|}{$2^{\text {nd }}$ reading of observer (1) } & \multirow[t]{2}{*}{$P$ value } \\
\hline & Number & $\%$ & Number & $\%$ & \\
\hline No osseous change & 2 & 10 & 2 & 10 & \\
\hline Condylar erosion & 12 & 60 & 9 & 45 & $* 0.034$ \\
\hline Condylar flattening & 4 & 20 & 6 & 30 & 0.102 \\
\hline Decreased Joint Space & 2 & 10 & 3 & 15 & 0.285 \\
\hline Total number & 20 & 100 & 20 & 100 & \\
\hline
\end{tabular}

TABLE (4) Intra-observer reliability of the CBCT observer (2)

\begin{tabular}{|c|c|c|c|c|c|}
\hline \multirow{2}{*}{ Types of observations } & \multicolumn{2}{|c|}{$1^{\text {st }}$ reading of observer (2) } & \multicolumn{2}{|c|}{$2^{\text {nd }}$ reading of observer (2) } & \multirow[t]{2}{*}{$P$ value } \\
\hline & Number & $\%$ & Number & $\%$ & \\
\hline No osseous change & 6 & 30 & 2 & 10 & $* 0.0001$ \\
\hline Condylar erosion & 6 & 30 & 12 & 60 & $* 0.0001$ \\
\hline Condylar flattening & 6 & 30 & 4 & 20 & 0.102 \\
\hline Decreased Joint Space & 2 & 10 & 2 & 10 & \\
\hline Total number & 20 & 100 & 20 & 100 & \\
\hline
\end{tabular}


TABLE (5) Inter-observer reliability of the 1st reading

\begin{tabular}{|c|c|c|c|c|c|}
\hline \multirow{2}{*}{ Type of observations } & \multicolumn{2}{|c|}{$1^{\text {st }}$ reading of observer $(1)$} & \multicolumn{2}{|c|}{$1^{\text {st }}$ reading of observer $(2)$} & \multirow{2}{*}{ P value } \\
\cline { 2 - 6 } & Number & $\%$ & Number & 30 & $* 0.0001$ \\
\hline No osseous change & 2 & 10 & 6 & 30 & $* 0.0001$ \\
\hline Condylar erosion & 12 & 60 & 6 & 30 & 0.102 \\
\hline Condylar flattening & 4 & 20 & 2 & 10 & \\
\hline Decreased Joint Space & 2 & 10 & 20 & 100 & \\
\hline Total number & 20 & 100 & & \\
\hline
\end{tabular}

TABLE (6) Inter-observer reliability of the $2^{\text {nd }}$ reading

\begin{tabular}{|c|c|c|c|c|c|}
\hline \multirow{2}{*}{ Type of observations } & \multicolumn{2}{|c|}{$2^{\text {nd }}$ reading of observer $(1)$} & \multicolumn{2}{|c|}{$2^{\text {nd }}$ reading of observer (2) } & \multirow{2}{*}{ P value } \\
\cline { 2 - 6 } & Number & $\%$ & Number & 10 & \\
\hline No osseous change & 2 & 10 & 2 & 60 & $* 0.034$ \\
\hline Condylar erosion & 9 & 45 & 12 & 20 & 0.102 \\
\hline Condylar flattening & 6 & 30 & 4 & 10 & 0.285 \\
\hline Decreased Joint Space & 3 & 15 & 2 & 100 & \\
\hline Total number & 20 & 100 & 20 & \\
\hline
\end{tabular}

\section{DISCUSSION}

CBCT provides low radiation dose, small equipment and has the ability to provide multiplanar reformation as well as 3D images. ${ }^{3}$ That's why this animal study was performed on an attempt to evaluate the diagnostic performance of CBCT for TMJ-OA in relation to histological examinations using $\mathrm{H} \& \mathrm{E}$ sections.

Adjuvant-induced arthritis (AIA) rat model injected by CFA inflammatory substance was performed on the study group of this work. Bendele et al ${ }^{5}$ proved that CFA accelerates and increases the incidence of arthritis resulting in acute periarticular inflammation followed by a phase of bone involvement. Spears et al ${ }^{6}$ as well as Kameoka et al ${ }^{4}$ also used CFA to study rat's OA. However, Ricks et al ${ }^{7}$ performed another rat TMJ-OA model (disproportionate micromelia "Dmm" rats) which exhibited similar characteristics of human OA.
On comparing histological and CBCT findings, the histological examination results confirmed arthritic changes of all the 20 condyles whereas CBCT detected changes of 19 condyle. Just one case in these results showed mild inflammation described histologically as a widening of marrow spaces, nonetheless, it was not detected by CBCT similar to the results by Ahliqvist and Isberg ${ }^{8}$ who suggested that CT detection of morphological changes less than $1 \mathrm{~mm}$ on temporal bone was very low. Moreover, Hussain et al ${ }^{2}$ mentioned that diagnostic imaging with $\mathrm{x}$-radiation needs at least $30 \%$ change in bone mineral content before it can be visualized on the radiographs.

Twelve cases showed no difference in condylar erosion findings. In these results, CBCT detected erosion as a surface irregularity or local bony defect similar to the results described by Katakami et al ${ }^{1}$ that the condylar erosion of cadaveric TMJ-OA was an irregular surface or thin bony cortex. 
Nevertheless, the histological analysis in the current study provided multiple explanations of the detected erosion such as surface irregularity, loss of cartilaginous cap, wide marrow spaces, bone marrow edema or hemorrhage which agrees with Katakami et al ${ }^{1}$ who referred histological erosion to decreased calcification and morphological changes of the bone cortex.

The 3D imaging of CBCT was suggested to be superior in the detection of condylar flattening in 5 out of 20 cases which couldn't be recognized precisely by plain 2D histological sections. Flattening was diagnosed histologically as a surface irregularity or loss of cartilaginous cap. However, Hintze et al ${ }^{9}$ had suggested that $23 \%$ only of condylar flattening were correctly identified by CBCT among TMJ-OA of dry skulls.

CBCT findings of decreased joint space were recorded in two cases out of 20 compared to four cases in histological analysis which may be attributed to the small sized rat's joint that complicated CBCT analysis.

Meanwhile, a study performed by Alexiou et al ${ }^{10}$ revealed that $\mathrm{CBCT}$ examination of human TMJ-OA detected $50 \%$ of the cases with decreased joint space and bone to bone contact in $22 \%$ of the examined patients.

Histological examination explained the reduced joint space as an inflammatory edema or proliferation of cartilaginous cap without actual bony outgrowth which disagrees with the results obtained by Flygare et al ${ }^{11}$ and Katakami et al ${ }^{1}$ who found hypercalcified regions and cartilaginous calcifications at the articulating surface of human condyles.

One of the arthritic changes described by Guerne et al ${ }^{12}$ was the proliferation of the synovial membrane into the condylar surface which was recorded in four cases in our results.

Regarding the diagnostic performance of CBCT, the sensitivity was $93 \%$. On the contrary, TMJ-OA of human dry skulls diagnosed in the studies by
Zain-Alabdeen and Alsahdan ${ }^{13}$ assumed low sensitivity (39.58\%) of CBCT while those obtained by Honda et al ${ }^{14}$ as well as Hussain et al ${ }^{2}$ recorded $80 \%$ sensitivity for CBCT. $100 \%$ specificity of CBCT was reported in this work which was similar to the results obtained by Honda et al ${ }^{14}$ on comparing CBCT with helical CT.

In addition, 95.29\% diagnostic accuracy for CBCT was proved to be similar to Alkhadr et al ${ }^{15}$ results and opposing to that of Hussain et al ${ }^{2}$ who reported $90 \%$ accuracy upon diagnosing TMJOA of human dry skulls. Positive predictive value (PPV) of CBCT was $100 \%$ and the same value was reported by $\mathrm{Nah}^{16}$ and Barghan et al ${ }^{17}$. However, negative predictive value (NPV) was $86 \%$ unlike to studies done by Nah ${ }^{16}$ and Barghan et al ${ }^{17}$ who detected $78 \%$ NPV.

Owing to the unfamiliar rat's anatomy as well as the small sized condyles, CBCT observers differed significantly between both observers at condylar erosion and no osseous changes findings resulting in insufficient reliability of CBCT readings for diagnosis of rat TMJ-OA in our results. Flygare et al ${ }^{11}$ explained that mild changes are difficult to visualize radiographically thus making the images more challenging and providing less accurate identification. However studies performed on human TMJ by Hintze et al ${ }^{9}$, Alkhader et al, ${ }^{15}$ and Zain-Alabdeen and Alsadhan ${ }^{13}$ confirmed high inter and intra observer reliability of CBCT for diagnosis of TMJ-OA.

In conclusion, CBCT was proved to have high diagnostic performance in detection of rat's condylar arthritis.

\section{REFERENCES}

1. Katakami K, Shimoda S, Kobayashi K, Kawasaki K. Histological investigation of osseous changes of mandibular condyles with backscattered electron images. Dentomaxillofac Radiol. 2008; 37:330-339

2. Hussain AM, Packota G, Major PW, Flores-Mir C. Role of different imaging modalities in assessment of temporomandibular joint erosions and osteophytes: a systematic review. Dentomaxillofac Radiol. 2008; 37:63-71 
3. Farman AG, Levato CM, Scarfe WC, Mah J. Education in the round: multidimensional imaging in dentistry. Inside Dent. 2008; 1:39-41

4. Kameoka S, Kuroki Y, Honda K, Kijima N, Matsumoto K, Asano M, Arai Y, Shirakawa T. Diagnostic accuracy of microcomputed tomography for osseous abnormalities in the rat temporomandibular joint condyle. Dentomaxillofac Radiol. 2009; 38:465-469

5. Bendele AM. Animal models of rheumatoid arthritis. J Musculoskel Neuron Interact. 2001; 4:377-385

6. Spears R, Dees LA, Sapozhnikov M, Bellinger LL, Hutchins B. Temporal changes in inflammatory mediator concentrations in an adjuvant model of temporomandibular joint inflammation. J Orofac Pain. 2005; 19:34-40

7. Ricks ML, Farrell TJ, Falk DJ, Holt DW, Rees M, Carr J, Williams T, Nichols BA, Bridgewater LC, Reynolds PR, Kooyman DL, Seegmiller RE. Osteoarthritis in temporomandibular joint of Col2a1 mutant mice. Arch Oral Biol. 2013; 58:1092-1099

8. Ahliqvist JB, Isberg AM. Validity of computed tomography in imaging thin walls of tempromporal bone. Dentomaxillofac Radiol. 1999; 28:13-19

9. Hintze H, Wiese M, Wenzel A. Cone beam CT and conventional tomography for the detection of morphological temporomandibular joint changes. Dentomaxillofac Radiol. 2007; 36:192-197

10. Alexiou KE, Stamatakis HC, Tsiklakis K. Evaluation of the severity of temporomandibular joint osteoarthritic changes related to age using cone beam computed tomography. Dentomaxillofac Radiol. 2009; 38:141-147
11. Flygare L, Rohlin M, Akerman S. Microscopy and tomography of erosive changes in the temporomandibular joint. An autopsy study . Acta Odontol Scand.1995; 53:297-303

12. Guerne P, Zuraw BL, Vaughan JH, Carson DA, Lotz M. Synovium as a source of interleukin 6 in vitro. Contribution to local and systemic manifestations of arthritis. J Clin Invest. 1989; 83:585-592

13. Zain-Alabdeen EH, Alsadhan RI. A comparative study of accuracy of detection of surface osseous changes in the temporomandibular joint using multidetector CT and cone beam CT. Dentomaxillofac Radiol. 2012; 41:185-191

14. Honda K, Larheim TA, Maruhashi K, Matsumoto K, Iwai $\mathrm{K}$. Osseous abnormalities of the mandibular condyle: diagnostic reliability of cone beam computed tomography compared with helical computed tomography based on an autopsy material. Dentomaxillofac Radiol. 2006; 35:152-157

15. Alkhader M, Ohbayashi N,Tetsumura A, Nakamura S, Okochi K, Momin MA, Kurabayashi T. Diagnostic performance of magnetic resonance imaging for detecting osseous abnormalities of the temporomandibular joint and its correlation with cone beam computed tomography. Dentomaxillofac Radiol. 2010; 39:270-276

16. Nah KS. Condylar bony changes in patients with temporomandibular disorders: a CBCT study. Image Sci Dent. 2012; 42:249-253

17. Barghan S, Tetradis S, Mallya SM. Application of cone beam computed tomography for assessment of the temporomandibular joints. Aust Dent J. 2012; 57:109-118 